Lucrările Seminarului Geografic Dimitrie Cantemir

Vol. 44, October 2017, pp. 69-84

http://dx.doi.org/10.15551/lsgdc.v44i0.05

\title{
La gouvernance publique/privée est-elle source d'innovation dans les territoires ruraux et périurbains ? L'exemple de la démarche leader
}

\section{Laurent Barbut ${ }^{1}$, Anne Desgree ${ }^{1}$, Jean-Philippe Housse ${ }^{1}$}

${ }^{1}$ EPICES - Évaluer les Politiques \& Innover pour les Citoyens et les Espaces, Paris, France

To cite this article: Barbut, L., Desgree, A., Housse, J.-P. (2017). La gouvernance publique/privée est-elle source d'innovation dans les territoires ruraux et périurbains?

L'exemple de la démarche leader. Lucrările Seminarului Geografic Dimitrie Cantemir, Vol. 44, pp. 69-84. DOI: $\underline{10.15551 / 1 \mathrm{sgdc} . v 44 \mathrm{i} 0.05}$

To link to this article: http://dx.doi.org/10.15551/lsgdc.v44i0.05 


\title{
LA GOUVERNANCE PUBLIQUE/PRIVÉE EST-ELLE SOURCE D'INNOVATION DANS LES TERRITOIRES RURAUX ET PÉRIURBAINS ? L'EXEMPLE DE LA DÉMARCHE LEADER
}

\author{
Laurent Barbut $^{1}$, Anne Desgree ${ }^{2}$, Jean-Philippe Housse ${ }^{3}$
}

\begin{abstract}
Résumé : La communication proposée s'appuie sur la démarche LEADER, en tant qu'exemple de politique publique impulsant et favorisant une gouvernance associant acteurs publics et privés dans les territoires ruraux et périurbains. A partir de plusieurs travaux d'évaluation et d'accompagnement de territoires dans la mise en œuvre de LEADER, nous chercherons à décrire comment les acteurs locaux répondent à cette impulsion et s'organisent pour mettre en œuvre une gouvernance publique/privée. Nous chercherons ensuite à évaluer dans quelle mesure cette nouvelle gouvernance génère ou non de l'innovation, que ce soit dans la gouvernance elle-même ou dans les projets émergents sur le territoire.

A partir de ce bilan de la mise en œuvre de cette forme de «nouvelle gouvernance », et de l'analyse des innovations qu'elle produit dans les territoires ruraux et périurbains, on tentera enfin de discuter les liens entre cette innovation principalement organisationnelle et le caractère durable du développement territorial.
\end{abstract}

\section{Introduction}

Le programme européen LEADER (Liaisons Entre Actions de Développement de l'Economie Rurale) s'inscrit clairement dans le champ du développement territorial durable des zones rurales et périurbaines, car il repose notamment sur la valorisation des ressources endogènes des territoires. Nous nous y intéressons ici en tant que politique publique impulsant et favorisant une gouvernance associant acteurs publics et privés, que l'on peut considérer a priori comme novatrice mais dont nous chercherons à analyser le caractère réellement innovant pour les territoires dans lesquels elle s'inscrit, parce que la mise en place de formes innovantes de gouvernance est considérée comme un facteur clé pour se rapprocher d'un développement territorial durable. Après avoir détaillé dans une première partie le cadrage théorique et la problématique de cette communication, nous en expliciterons les sources dans une deuxième partie et en présenterons les principaux résultats dans une troisième partie, avant d'ouvrir quelques pistes de discussions et de perspectives.

\footnotetext{
${ }^{1}$ directeur, EPICES (46 rue Bichat 75010 PARIS, www.epices-net.fr), laurent.barbut@epices-net.fr

2 consultante associée, EPICES

${ }^{3}$ chargé d'études, EPICES
} 


\section{Cadrage théorique}

\subsection{Développement Territorial Durable, gouvernance et innovation}

La «mise en territoire» du développement durable — ou la recherche d'un Développement Territorial Durable (DTD) - implique de repenser les périmètres de l'action publique et requiert de nouveaux modes de gouvernance, et à ce titre implique des démarches résolument innovantes. Définir des moyens et un nouveau périmètre d'action nécessite en effet l'intervention sur la scène du développement local d'acteurs de plus en plus nombreux et diversifiés, qu'il convient d'identifier, de mobiliser et de coordonner (Carrière et al., 2016). Le DTD, parce qu'il renvoie à des problèmes complexes, conflictuels et controversés, dont la solution passe par la mobilisation d'acteurs nombreux, interagissant à diverses échelles, et dont les territoires ne correspondent généralement pas aux territoires institutionnels classiques, est logiquement un «laboratoire » de production de ces nouvelles formes de gouvernance. Néanmoins, pour de nombreux observateurs, les résultats de ces nouvelles formes de gouvernance sont décevants - par exemple en termes de mobilisation de la société civile - sans qu'il soit facile de juger si ces défaillances sont imputables à une gouvernance mal conçue... ou au contraire à un déficit de gouvernance. C'est d'autant plus problématique que la «bonne » gouvernance nécessaire au DTD suppose des changements structurels et un ensemble d'innovations qui vont manifestement très au delà du bricolage pragmatique et des «arrangements de terrain » dans lesquels on la cantonne généralement (Theys, 2002). La capacité des nouvelles formes de gouvernance territoriale à être véritablement porteuses d'innovation est donc à la fois un enjeu et un questionnement central du Développement Territorial Durable.

\subsection{Les nouvelles formes de gouvernance territoriale reposant sur du partenariat public privé (PPP) sont-elles porteuses d'innovation ?}

Le concept de gouvernance territoriale trouve ses origines à la fois dans l'économie et la science politique (Leloup et al., 2005). Le terme de gouvernance apparaît il y a plus d'un demi siècle chez les économistes qui s'intéressent aux coordinations " hors marché » entre agents individuels et collectifs (école des coûts de transaction). Puis, à la fin des années 1980, le terme est importé dans les sciences politiques pour caractériser les modalités de gouvernement régissant les agglomérations (en particulier métropolitaines) et, par extension, l'action publique territoriale. Dans le cadre de cette analyse du gouvernement local, l'usage du concept de gouvernance entend souligner la participation de divers intérêts, notamment privés, aux systèmes de décision publique qui régissent les villes et les régions. Cette introduction de la notion de gouvernance en sciences politiques traduit la reconfiguration de l'action publique, l'émergence de nouveaux modes d'intervention et la transformation des modalités de l'action publique. Elle repose sur la dénonciation du modèle de politique traditionnel, descendant et centralisé. Elle met l'accent sur la multiplicité et la variété des acteurs (ONG, entreprises privées, citoyens,) associés à la définition et à la mise en œuvre de l'action publique. Le point commun des diverses approches de la notion de gouvernance réside dans la prise en compte de l'élargissement du champ des acteurs impliqués, de l'interdépendance des acteurs et des organisations tant privées que publiques dans le processus de prise de décision (Leloup et al., 2005). Dans le cadre de l'approche du territoire 
défini comme une construction d'acteurs, développée parallèlement par plusieurs auteurs (Pecqueur, 2002), la gouvernance territoriale est comprise comme un processus non seulement de coordination des acteurs mais aussi d'appropriation des ressources et de construction de la territorialité (Leloup et al., 2005).

La mise en œuvre pratique de ce processus de gouvernance territoriale a fait l'objet de plusieurs analyses. Bertrand et Moquay observent ainsi plusieurs formes d'incitations publiques favorisant la mise en place de ces nouvelles formes de gouvernance (procédures contractuelles, réseaux de villes, conseils de quartiers,...). Ils en déduisent un décalage entre les principes des politiques qui se réclament de la gouvernance et leur mise en œuvre pratique, qui s'explique assez largement par le fait que, contrairement aux institutions formelles, la gouvernance locale se construit, mais ne se décrète pas (Bertrand et Moquay, 2004).

D'autres observateurs de ces nouvelles coordinations entre acteurs publics et privés rejoignent cette conclusion, et soulignent que ces «constructions » peuvent donner lieu à de multiples «bricolages institutionnels », où les acteurs sociaux mobilisent et exploitent les «moyens du bord » et ressources à leur disposition, les détournant de leur finalité première pour reconstruire de nouvelles institutions. Ces compromis émergents constituent, toujours selon ces mêmes auteurs, une source d'innovation institutionnelle particulièrement intéressante (Xhauflair et al., 2010).

Si l'on se réfère aux récents efforts de définition de la notion d'innovation territoriale — « Sous la catégorie d'innovation territoriale sont classées toutes les initiatives identifiées sur un territoire à caractère de préférence novateur ou inhabituel pour le territoire » (DIACT et ARF, 2009) - la fabrique de nouveaux dispositifs de coordination semble effectivement contribuer assez directement à l'innovation territoriale. Pour autant, les observateurs de ces démarches de nouvelle gouvernance territoriale insistent également, voire davantage, sur les freins existants au développement de cette innovation : difficultés à mobiliser les acteurs locaux, temps nécessaire à la construction d'un capital confiance, risque de «normalisation » des processus en cas de généralisation de l'incitation à la mise en place de cette nouvelle gouvernance par les politiques publiques (Chia et al., 2010 ; Bertrand et Moquay, 2004 ; Doré, 2011).

Nous nous inscrivons dans ce champ d'analyse issu du constat que, sous l'injonction de nombreuses politiques publiques, les acteurs locaux privés sont de plus en plus sollicités pour participer aux processus de décision et de mise en place des actions concernant la gestion des territoires. Nous constatons comme les auteurs cités plus haut que, dans ces situations, on peut observer des pratiques mises en œuvre pour fabriquer de nouveaux dispositifs permettant d'assurer la coordination entre les acteurs publics et privés au sein des territoires. Mais ces nouvelles pratiques sont-elles vraiment porteuses d'innovation ? Si oui, quelles sont les formes que prend cette innovation: organisationnelle, technique, commerciale? à l'échelle de la gouvernance du territoire et/ou au sein des entreprises publiques et privés qui le compose? Peut-on favoriser ces pratiques innovantes ou les généraliser au service d'un développement plus durable des territoires ? Une fois apparues, peut-on favoriser leur ancrage dans la durée ou restent-elles fortement tributaires d'une incitation financière et/ou de l'implication de certaines personnalités influentes ? Autant de questions que nous chercherons à éclairer dans la suite de cette communication en nous appuyant principalement sur l'exemple de la politique LEADER. 


\subsection{Le programme LEADER, une injonction à la mise en place de PPP dans la gouvernance des territoires ruraux et périurbains}

Le programme européen LEADER (Liaisons Entre Actions de Développement de l'Economie Rurale) a été lancé en 1991 par la Commission Européenne dans un souci de diversification de l'économie des zones rurales. L'approche prônée par ce programme est généralement résumée au travers de sept principes de mise en œuvre, parmi lesquels (1) un partenariat local public privé de décision, formalisé au sein d'un Groupe d'Action Locale (GAL) dans lequel les acteurs privés ont une place majoritaire, et (2) l'innovation entendue au sens large (nouveau produit, nouveau processus, nouvelle organisation, nouveau marché...). Au cours des quatre générations de programmes LEADER qui se sont succédées depuis $1991^{4}$, et qui ont abouti à une certaine forme de généralisation de cette approche au sein des territoires ruraux et périurbains français et européens, ces principes n'ont pas ou peu évolué.

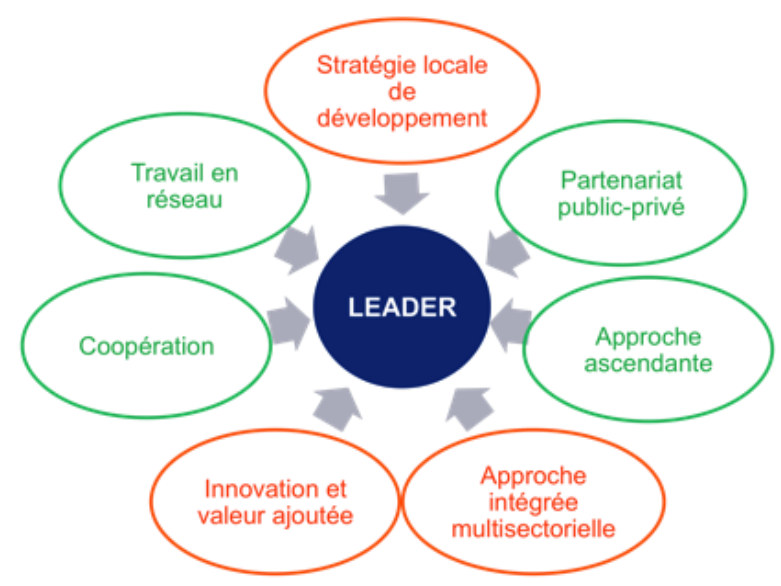

Figure 1 : Les 7 principes de mise en œuvre de l'approche LEADER

Le programme LEADER s'appuie donc sur un groupe d'action locale (GAL) associant acteurs publics et privés qui étudie les besoins spécifiques du territoire, les débat de manière ouverte et définit une stratégie locale de développement visant à appuyer les initiatives locales par un soutien technique et/ou financier aux porteurs de projet. LEADER vient en complémentarité des fonds locaux procurant un effet levier aux politiques publiques locales. Les pratiques expérimentées localement ont vocation à être diffusées aux autres territoires ruraux. Le programme LEADER s'adresse prioritairement à de «petits » porteurs de projets issus de la société civile développant, selon une logique ascendante, dans l'espace rural, à l'appui de ressources locales originales, des projets transversaux à caractère innovant permettant le développement de dimensions ciblées du territoire. Il s'agit bien de «faire

\footnotetext{
${ }^{4}$ LEADER I de 1991 à 1994 (40 GAL en France), LEADER II de 1995 à 2000 (179 GAL en France), LEADER + de 2000 à 2006 (140 GAL en France) et Programme LEADER 2007-2013 (222 GAL en France).
} 
émerger» des projets «avant-gardistes» qui explorent de nouvelles approches de développement (Mathé et al., 2014). On notera à travers cette description l'imbrication des deux principes de mise en œuvre de LEADER qui nous intéressent particulièrement ici : le PPP est à la fois une innovation organisationnelle (nouvelle gouvernance) et une source d'innovation à travers les projets « avant-gardistes » qu'il est censé permettre de repérer et de financer.

Le programme LEADER constitue donc pour les territoires ruraux une incitation puissante - à travers les financements qu'il permet d'obtenir — et une forte injonction — à travers les principes à respecter pour y accéder - à mettre en place une gouvernance locale associant acteurs publics et acteurs privés. De plus, l'existence au sein du programme LEADER de quelques critères d'éligibilité précis et de nature relativement aisément vérifiable, renvoyant à l'application des principes sur lequel le programme repose, favorise leur mise en œuvre effective. En ce qui concerne le PPP notamment, les règlements stipulent qu'une instance de décision locale doit être mise en place pour élaborer la stratégie, mettre en place une procédure de soumission des projets, réceptionner et évaluer les demandes de soutien et suivre l'application de la stratégie locale de développement, et qu'au sein de cette instance, ni les autorités publiques, ni un groupement d'intérêt ne représentent plus de $49 \%$ des droits de vote.

Enfin, à travers ses programmes successifs depuis plus de 20 ans, et l'élargissement $\mathrm{du}$ nombre de territoires qui ont pu en bénéficier, cette incitation/injonction concerne aujourd'hui un nombre important de territoires, ayant expérimenté la mise en œuvre de cette nouvelle gouvernance depuis plus ou moins longtemps. C'est pour ces différentes raisons que nous considérons que LEADER est un bon exemple pour observer et analyser la mise en œuvre locale de nouvelles formes de gouvernance reposant sur le PPP.

La problématique que nous nous proposons de traiter dans la suite de cette communication est donc la suivante: analyser, à partir de l'exemple du programme LEADER dont on fait l'hypothèse qu'il constitue une injonction à la mise en place d'une gouvernance innovante, la réalité de cette innovation dans ses différentes dimensions : innovation organisationnelle (gouvernance territoriale, nature des bénéficiaires de l'action publique), innovation dans les projets menés sur le territoire. Puis, suite à cette analyse, tenter de conclure sur les liens entre cette innovation et le caractère durable du développement territorial.

\section{Sources et méthodologie}

L'analyse que nous proposons dans cette communication de la mise en œuvre effective du PPP dans les territoires ruraux bénéficiant — ou cherchant à bénéficier — du programme LEADER s'appuie sur un certain nombre de missions d'évaluation ou d'accompagnement conduites par les auteurs. Elle émane de praticiens de l'accompagnement des démarches territoriales, qui se situent ici dans une posture de «participation observante ». Les travaux plus précisément mobilisés sont les suivants : 


\section{a) l'évaluation finale du programme LEADER 2007-2013 dans une région française}

Réalisée en 2013-2014 à la demande des 5 GAL de la région Champagne Ardenne ${ }^{5}$ ayant bénéficié du programme LEADER 2007-2013 cette évaluation finale présente l'originalité d'avoir fait l'objet d'un projet de coopération entre les 5 GAL. Ce choix s'est traduit par une forte implication des cinq territoires dans la collecte et le traitement des informations, ayant permis une analyse très approfondie à la fois de la gouvernance et des résultats du programme. Sur le plan de la gouvernance, qui nous intéresse plus particulièrement ici, on peut noter qu'environ la moitié des membres des 5 comités de programmation $^{6}$ ont été interrogés (soit environ 60 personnes), et que de nombreuses données factuelles sur le fonctionnement concret des instances décisionnelles ont pu être rassemblées (nombre de réunions, assiduité des acteurs publics et privés, temps de débat, modalités de vote, ...). Enfin, une enquête a été conduite auprès de l'ensemble des porteurs de projets sur les cinq territoires, qui a permis d'obtenir 115 réponses.

b) l'accompagnement des 18 territoires de la région Champagne Ardenne pour l'élaboration de leur candidature au programme LEADER 2014-2020

Effectué au cours de l'année 2015, cet accompagnement comporte à la fois une dimension individuelle (appui spécifique de chaque territoire candidat) et une dimension collective (réalisation de séminaires et de journées de formation pour l'ensemble des 18 territoires candidats). Dans la mesure où le PPP doit être mis en place dès l'élaboration de la candidature, et que cette mise en place effective constitue un critère de sélection important pour bénéficier du programme ${ }^{7}$, cette mission d'accompagnement constitue un excellent «poste d'observation » de territoires confrontés, pour la plupart pour la première fois ${ }^{8}$, à la nécessité de modifier leurs pratiques habituelles de gouvernance.

\section{Principaux résultats}

\subsection{L'injonction à mettre en place une gouvernance PPP ne révèle pas un fort potentiel d'innovation institutionnelle}

Afin d'accéder au programme LEADER, puis pour le mettre en œuvre, les territoires ruraux et périurbains sont donc confrontés à la nécessité de mettre en place une gouvernance associant acteurs publics et acteurs privés. Au delà de cette injonction de principe et de quelques critères précis permettant d'en vérifier l'application effective (constitution d'une instance décisionnelle dans laquelle les acteurs privés sont majoritaires), les territoires se retrouvent alors face à un certain nombre de questions à résoudre pour mettre en œuvre

\footnotetext{
${ }^{5}$ Suite à la réforme intervenue fin 2015, la région Champagne Ardenne est désormais intégrée à la région Grand Est.

${ }^{6}$ Le comité de programmation est l'instance décisionnelle du GAL, dans laquelle les acteurs privés doivent représenter au moins $51 \%$ des droits de vote.

${ }^{7}$ L'accession au programme LEADER s'effectue sous forme d'un appel à candidature, suivi d'une procédure de sélection basée notamment sur le respect des principes de la démarche LEADER.

${ }^{8}$ Nous avons indiqué plus haut que seuls 5 territoires bénéficiaient du programme LEADER en Champagne Ardenne pour la période 2007-2013. Sachant qu'ils ont tous choisi de faire une nouvelle candidature au programme 2014-2020, et qu'il y a 18 candidats, 13 territoires candidatent donc pour la première fois.
} 
concrètement ce qui leur est proposé. Nous examinerons particulièrement ici les réponses apportées à quatre de ces questions, qui nous paraissent parmi les plus centrales :

- comment repérer des acteurs privés pour construire un PPP avec eux sur le programme LEADER ?

- comment entretenir la mobilisation des acteurs du PPP, privés mais aussi publics, pendant plusieurs années, afin que le partenariat reste effectif ?

- comment s'assurer que les acteurs privés participant sont représentatifs des forces économiques et sociales du territoire ?

- comment, enfin, gérer les inévitables conflits d'intérêt et asymétries de pouvoir que va occasionner la gestion d'un programme comme LEADER en ayant recours au PPP?

\subsubsection{Le repérage des acteurs}

Concernant cette première question, ou ce premier enjeu, il convient tout d'abord de distinguer deux situations dans les territoires qui y sont confrontés, selon la préexistence ou non de forme organisée d'implication de la société civile dans la gouvernance territoriale. Dans le cas de LEADER que nous traitons ici, cette forme organisée éventuellement préexistante peut être un conseil de développement de Pays ou des commissions impliquant les acteurs dans la gouvernance d'un PNR ${ }^{9}$. Notons d'emblée que ces deux situations semblent assez polaires (existence ou non existence), mais que la réalité de la gouvernance territoriale fait que ce n'est souvent pas si tranché (de telles instances existent « sur le papier » mais elles ne se réunissent plus, ou très peu souvent, etc.).

Considérons donc en premier lieu les situations où il n'existe pas de telles formes organisées d'implication de la société civile, qui sont le signe d'une gouvernance territoriale dans laquelle les élus maîtrisent mal le PPP et n'ont pas ou peu l'habitude de travailler avec les acteurs privés de leur territoire. Face à la nécessité de repérer et de mobiliser des acteurs privés pour monter ou gérer un programme LEADER, les acteurs publics des territoires que nous avons pu observer dans le cadre des travaux mentionnés ci-dessus empruntent généralement deux types de voies, éventuellement combinables :

- la première, que nous pourrons qualifier de plutôt «inductive », consiste à organiser une ou plusieurs réunions publiques ouvertes à l'ensemble des acteurs du territoire, à présenter lors de ces réunions le programme LEADER et ses principes, et à «recruter» dans le cadre de ces réunions des acteurs privés volontaires pour participer à la gouvernance du programme. Si cette première voie bénéficie a priori d'une grande ouverture à l'ensemble des acteurs du territoire, elle n'est pas exempte de risques : biais de sélection à travers la diffusion de l'information sur l'existence des réunions publiques (seuls les acteurs au courant de ces réunions peuvent y participer), risque d'avoir une majorité de volontaires ne représentant «qu'eux mêmes » et donc pas nécessairement les forces économiques et sociales du territoire ;

\footnotetext{
${ }^{9}$ Les structures sur lesquelles s'appuient généralement les programmes LEADER en France sont des Pays ou des Parcs Naturels Régionaux (PNR).
} 
- la deuxième voie, que nous pourrions qualifier de plus « déductive » ou de plus construite, consiste à s'appuyer sur les connaissances informelles des acteurs publics pour identifier, par itération successive, des acteurs privés répondant au profil recherché, et ensuite les solliciter pour leur proposer d'intégrer la gouvernance du programme LEADER. Cette deuxième voie permet de laisser moins de place au hasard, mais présente le risque de la «dépendance au sentier ", c'est-à-dire de se tourner en premier lieu vers les représentants organisés de la société civile qui sont les interlocuteurs « habituels » des acteurs publics (chambres consulaires notamment). Or ces acteurs n'ont pas nécessairement de forte légitimité territoriale (ils sont structurés à l'échelle départementale).

Considérons maintenant le cas où une forme organisée d'implication de la société civile préexiste sur le territoire (conseil de développement notamment). La démarche « naturelle » pour organiser la gouvernance du programme LEADER est alors de proposer à cette instance de désigner un certain nombre d'acteurs privés — parmi ses membres ou audelà - pour intégrer l'instance de pilotage. Cela revient à considérer que cette instance préexistante a déjà acquis une forme de légitimité à représenter la société civile du territoire et que l'on peut s'appuyer sur cet acquis pour définir la gouvernance LEADER. Le problème est ici que, comme nous l'indiquions plus haut, cette légitimité est parfois très relative (en cas d'essoufflement de cette instance depuis plusieurs années par exemple). Les acteurs sont alors confrontés à un dilemme : s'appuyer sur une organisation peu légitime et risquer une désignation peu satisfaisante, ou la «contourner » et risquer alors de se «mettre à dos » les membres de cette organisation ${ }^{10}$. Nos observations montrent que face à ce dilemme, les choix des acteurs se portent vers l'une ou l'autre des solutions, voire sur une combinaison des deux.

\subsubsection{La mobilisation des acteurs dans la durée}

L'enjeu de la mobilisation des acteurs dans la durée est central pour toutes les démarches de gouvernance territoriale reposant sur du PPP. Soulignons tout d'abord qu'en déléguant à l'échelle territoriale la responsabilité de sélectionner les projets à financer, le programme LEADER permet de gérer plus facilement cet enjeu car l'instance décisionnelle a des choix à faire tout au long de la durée du programme. En effet, les entretiens qualitatifs menés auprès de nombreux membres de comités de programmation LEADER soulignent l'importance, en termes de mobilisation des acteurs, du maintien dans la durée du sentiment de participer à une instance ayant une véritable responsabilité décisionnelle.

Le pendant de cette responsabilisation des acteurs est bien entendu l'investissement nécessaire que cela entraîne, et qui se heurte, pour les acteurs privés notamment, aux contraintes de temps pour réaliser cet investissement dans la durée. Nos observations soulignent diverses tentatives des territoires pour s'adapter au mieux à ces contraintes : réunions en soirée, réunions «tournantes » sur différentes localisations au sein du territoire, recherche de convivialité, etc. Ces diverses formes d'organisation logistique apparaissent

${ }^{10}$ Risque qu'il ne faut pas négliger dans le contexte, rappelons-le, de territoires de taille relativement modeste, dans lesquels les acteurs constituant des forces vives ne sont pas nécessairement très nombreux. 
plutôt bienvenues, mais ne sont pas déterminantes pour l'enjeu de la mobilisation (en raison, notamment, de la diversité des situations individuelles à concilier).

Un facteur plus important pour l'enjeu de mobilisation des acteurs dans la durée réside en revanche dans la capacité à acquérir une forme de compréhension, ou de culture, vis-à-vis d'un programme public comme LEADER, que l'on peut considérer à bien des égards comme relativement complexe (nombreuses échelles territoriales impliquées, nombreux principes de mise en œuvre, nombreuses contraintes techniques de gestion, etc.). A cet égard, l'organisation de formations à destination des membres des comités de programmation, notamment peu après la mise en place de ces instances, ou leur renouvellement, apparaît comme une pratique très intéressante.

Enfin, soulignons que l'enjeu de mobilisation des acteurs dans la durée ne concerne pas seulement les acteurs privés, et que, s'agissant des élus, les fréquents «cumuls de mandat $\gg$ ne constituent pas un facteur favorable.

\subsubsection{La question de la représentativité}

Le troisième enjeu auquel sont confrontés les acteurs cherchant à mettre en place une gouvernance territoriale reposant sur du PPP est celui de la représentativité des acteurs privés au regard de la population locale. Cette question, qui ne se pose pas dans les mêmes termes pour les acteurs publics ${ }^{11}$, renvoie au mode de désignation des représentants des acteurs privés (voir ci-dessus) et conditionne de façon importante la légitimité des décisions prises pour les élus d'une part, et pour les habitants du territoire, d'autre part. Par ailleurs, ce troisième enjeu est d'une certaine façon «en conflit » avec le précédent : en effet, c'est souvent parce qu'il est difficile de trouver des acteurs privés motivés dans la durée que l'on travaille «toujours avec les mêmes », au détriment de l'optimisation de la représentativité. La Cour des Comptes Européenne souligne ainsi dans un de ses rapports que très peu d'instances décisionnelles de GAL compte des jeunes parmi ses membres, alors que « les jeunes constituent un important groupe cible pour la politique de développement rural de l'UE et que leur participation au processus de prise de décision peut faire en sorte que leurs besoins et leurs perspectives soient dûment prise en compte et que des solutions appropriées soient mises en œuvre » (UE, 2010). Enfin, cette question de la représentativité des acteurs privés peut également se poser au regard de la mise en œuvre du principe de PPP. Le rapport de la Cour des Comptes Européenne cité précédemment pointe également le cas d'un GAL français dans lequel les membres du comité décisionnel issus du secteur privé étaient principalement des représentants d'associations créées par les pouvoirs publics locaux (UE, 2010). Le cas des représentants des Chambres Consulaires pose des questions du même ordre, la «convention » généralement adoptée en France étant de les considérer comme représentants du secteur privé s'il s'agit d'un représentant professionnel élu, et comme représentant du secteur public s'il s'agit d'un salarié de la structure.

Les initiatives que l'on a pu observer pour gérer cette question de la représentativité des acteurs privés restent rares, sans doute en raison du caractère surdéterminant de la difficulté de mobilisation. Au delà d'une approche empirique du « repérage » intégrant plus ou moins cette notion de représentativité (voir plus haut), on notera simplement l'exemple d'un territoire ayant mené des actions spécifiques auprès des jeunes de son territoire lors de

${ }^{11}$ Dans la mesure où ils sont soumis régulièrement à des procédures de vote. 
l'élaboration de sa candidature LEADER (participation des collégiens et lycéens au diagnostic de territoire).

\subsubsection{La gestion des conflits d'intérêt et asymétries de pouvoir}

A l'échelle d'un territoire rural ou périurbain bénéficiant d'un programme LEADER $^{12}$, la mise en place d'une instance associant acteurs publics et acteurs privés pour sélectionner les projets éligibles aux financements du programme introduit de fait la nécessité de gérer de potentiels conflits d'intérêts et asymétries de pouvoir. Ces derniers sont principalement de deux types :

- conflit d'intérêt entre un porteur de projet du territoire présentant un lien plus ou moins direct avec un membre de l'instance de décision ${ }^{13}$;

- asymétries de pouvoir «réel » entre des acteurs privés majoritaires en nombre au sein de l'instance décisionnelle et acteurs publics disposant pour certains d'un pouvoir important sur l'octroi de fonds nécessaires à la réalisation des projets soumis au comité.

La gestion de ce type d'enjeu peut se faire selon deux grandes modalités dans les territoires LEADER que nous avons pu observer. La première modalité est tout à fait informelle et ne peut se repérer qu'à l'occasion d'entretiens qualitatifs tels qu'ils sont menés dans une approche évaluative, c'est-à-dire avec l'assurance d'un traitement anonyme des réponses. Il s'agit notamment du choix de confier la présidence de l'instance décisionnelle à un acteur privé, avec «l'autorisation implicite » des acteurs publics influents, pour donner une image plus équilibrée des pouvoirs réels, et/ou servir de caution ou d'alibi selon les cas.

Le rééquilibrage réel des pouvoirs dépendra fortement dans une telle situation de la personnalité du Président (ou de la Présidente) choisi, et de sa capacité à «tenir tête » à un élu influent ayant une grande expérience de l'exercice du pouvoir ${ }^{14}$.

La seconde modalité de gestion des risques de conflits d'intérêt et asymétries de pouvoir est, à l'opposé de la précédente, l'instauration de règles de décision formelles permettant de gérer - sans les éliminer - les enjeux de ce type. Cette seconde modalité suppose une certaine prise de conscience de l'existence de ces risques, et donc une volonté explicite et discutée de les gérer, ce qui n'est pas toujours évident dans un contexte ou, rappelons-le, le volontariat est légion. En fonction du niveau de "prise de conscience» atteint par les territoires, on peut donc classer les règles adoptées en fonction de leur capacité croissante à limiter les risques de conflits d'intérêt et asymétries de pouvoir :

- une première règle, que l'on peut qualifier de «base», et de fait adoptée par quasiment tous les territoires, consiste à inscrire dans leur règlement intérieur - explicite ou implicite - le fait que tout membre ayant un lien avec un projet présenté ne participe pas au vote. L'effet de cette première procédure est fortement limité par l'usage, qui fait que de nombreux projets ne font pas l'objet

\footnotetext{
${ }^{12} 150000$ habitants au maximum.

${ }^{13}$ A titre d'exemple, certains de nos travaux d'évaluation ont montré que ce cas de figure représentait entre 30 et $60 \%$ des projets selon les territoires.

${ }^{14}$ Notre expérience montre qu'en la matière le domaine professionnel dans lequel exerce, ou a exercé, l'acteur privé est souvent déterminant (chef d'entreprise, proviseur de lycée,...).
} 
de votes formels mais sont sélectionnés à l'unanimité, éventuellement après discussion ;

- une deuxième règle, beaucoup plus rarement mise en œuvre ${ }^{15}$, consiste à effectuer des votes à bulletins secrets pour la sélection de chaque projet ;

- une troisième règle, enfin, consiste à instruire une grille de sélection pour chaque projet, sur la base de critères définis collectivement au préalable, et utiliser réellement cette grille pour la sélection des projets ${ }^{16}$.

Comme on le constate aisément, les règles ou les procédures les plus efficaces sont aussi les plus demandeuses de temps, ce qui souligne une fois encore le lien existant entre la qualité des processus de gouvernance et les moyens humains disponibles (animation salariée et engagement bénévole). On soulignera enfin que les processus de «prise de recul» sur la gouvernance, au premier rang desquels la pratique de l'évaluation externe ou de l'autoévaluation, sont de puissants moyens de prise de conscience collective de ces risques de conflits d'intérêt et d'asymétries de pouvoir, et favorisent donc grandement le renforcement des procédures et des règles mises en place pour les gérer.

\subsubsection{Conclusion}

A l'issue de cette analyse de la façon dont les acteurs s'organisent pour répondre à cette injonction (repérage des acteurs privés, entretien de leur mobilisation dans la durée, gestion de la représentativité, gestion des conflits d'intérêt et asymétries de pouvoir), on peut faire le constat que les réponses apportées par les acteurs territoriaux à l'injonction de mise en place d'une gouvernance reposant sur le PPP ne révèle pas un fort potentiel d'innovation institutionnelle. On observe en effet un certain nombre de « bricolages institutionnels », mais qui restent dans l'ensemble assez classiques et proches de ce que l'on peut trouver notamment dans la gouvernance associative. Cette conclusion soulève la question de savoir si ce résultat est dû à la politique analysée (LEADER) ou s'explique par des facteurs plus structurels. Sans qu'il soit possible de trancher tout à fait, on peut mettre en avant quelques hypothèses :

- au titre de LEADER, la complexité de la gestion de ce programme qui fait que les aspects «techniques» prennent vite le pas sur les débats de fond, et renforcent le pouvoir réel de la «technostructure » par rapport à la gouvernance publique/privée ;

- au titre des explications plus structurelles, les cumuls de mandats et les problèmes de disponibilités, des acteurs publics comme privés, qui freinent l'innovation dans la mise en place de ces nouvelles formes de gouvernance. Sans oublier bien sûr dans ces explications plus structurelles, la culture inhérente à ces nouveaux processus, dont le développement reste très lent...

\footnotetext{
${ }^{15}$ A titre d'exemple, l'évaluation menée sur les 5 GAL d'une région française a mis en évidence que cette modalité n'existait que dans un territoire sur cinq.

${ }^{16}$ La dernière partie de la phrase est importante car nous avons pu observer dans de nombreux cas qu'une telle grille existait (généralement conçue en début de programme), mais n'était pas ou plus utilisée.
} 


\subsection{En revanche ce type de gouvernance peut favoriser une distribution « atypique » des porteurs de projet soutenus}

Si l'on s'intéresse à présent à la nature des porteurs de projets soutenus par le programme LEADER, on constate une distribution des porteurs de projet soutenus assez nettement différente de celle que l'on observe avec les autres politiques et programmes de développement régional/territorial financé par l'UE (fonds structurel FEDER notamment). Le graphique ci-après souligne en effet (1) l'importance des porteurs de projets de nature associative dans les cinq GAL analysés, et (2) l'importance des particuliers dans deux territoires sur les cinq, alors que ces deux types de porteurs de projets sont généralement très peu présents dans les cibles des autres politiques et programmes de développement régional/territorial financé par l'UE.

Les travaux que nous avons pu réaliser permettent de relier cette distribution atypique des porteurs de projets soutenus à deux aspects importants des programmes LEADER :

- le premier est la capacité de ce programme à financer de l'animation au sein des territoires, qui permet d'accompagner des porteurs de projets disposant de peu de ressources administratives et financières dans le montage de projets relativement complexes, favorisant ainsi leur accession à des financements européens auxquels ils n'auraient pas eu accès sans cet accompagnement. Cette animation, si elle est un facteur d'explication important de la présence de nombreuses associations — dont bon nombre de petite taille — et de particuliers au sein des porteurs de projets financés par LEADER, n'est cependant pas le seul. En effet, d'une part, LEADER n'est pas le seul programme permettant de financer de l'animation territoriale, et d'autre part, tous les territoires LEADER bénéficiant d'animation ne présentent pas une distribution aussi atypique des porteurs de projets ;

- un deuxième facteur explicatif est donc à considérer, et il s'agit bien de la gouvernance particulière de LEADER reposant sur le PPP. Cette dernière influence de plusieurs façons la présence de porteurs de projets atypiques au sein des bénéficiaires du programme: d'une part, les acteurs privés présents dans l'instance décisionnelle diffusent auprès de leurs pairs la recherche de projets et la possibilité de bénéficier de soutiens européens (effet réseau), et, d'autre part, la présence majoritaire de ces mêmes acteurs privés au sein de l'instance attribuant les financements influence la nature des porteurs de projets retenus (effet démocratique). On peut d'ailleurs émettre l'hypothèse que cette influence de la gouvernance particulière de LEADER sur la répartition des porteurs de projets sera d'autant plus importante en fonction du degré d'innovation qu'elle aura généré pour sa mise en place. 


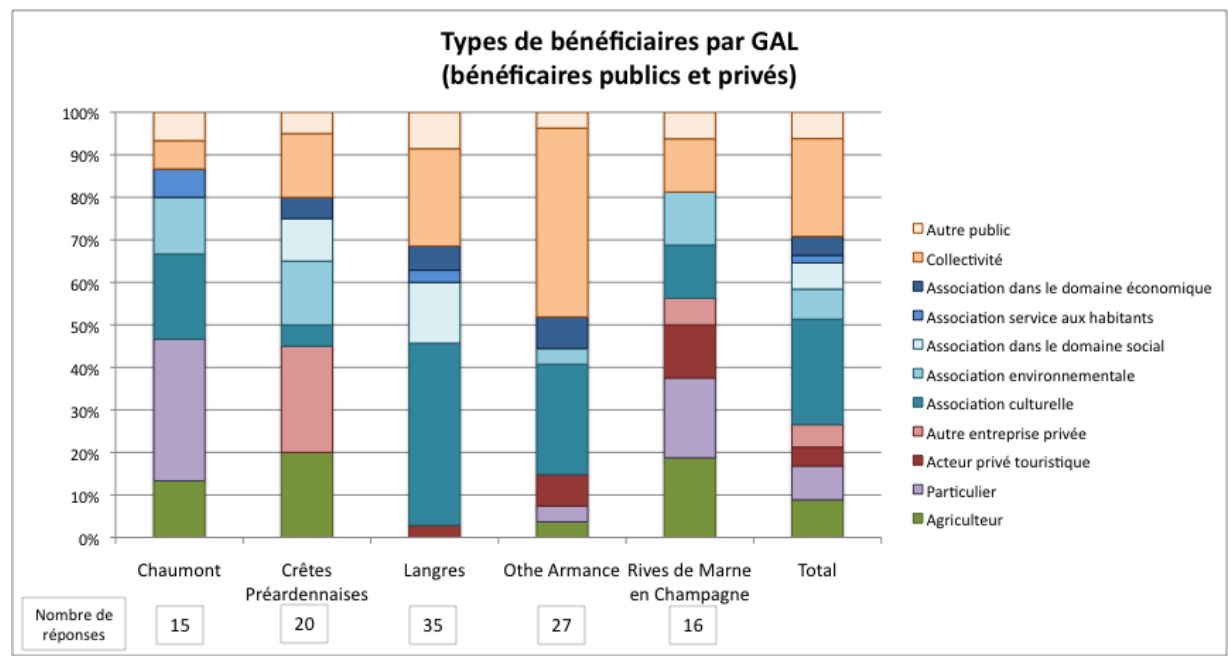

Figure 2 : Les types de bénéficiaires de GAL (bénéficiaires publics et prives)

Il convient néanmoins de rappeler ici que de nombreux auteurs spécialistes de LEADER considèrent que la capacité de ce programme à soutenir des porteurs de projets privés s'est affaiblie au cours des différentes générations de programme, ce constat valant encore plus pour les porteurs de projets «atypiques » de type associatif ou personne physique. Ces auteurs attribuent ce recul à l'augmentation progressive des contraintes administratives et financières pour accéder au financement, qui est effectivement indiscutable en ce qui concerne notamment la période 2007/2013 (Mathé, 2014 ; Vollet, 2017). Ce constat, que nous partageons, n'est pas contradictoire avec ce que nous avons montré plus haut. En effet (1) la proportion de bénéficiaires privés est effectivement variable dans les territoires que nous analysons, (2) nous faisons l'hypothèse qu'elle s'explique à la fois par une animation dynamique et une mise en œuvre active du PPP dans la gouvernance territoriale, conditions qui sont loin d'être réunies dans l'ensemble des territoires LEADER (notamment la seconde). Le lien entre une gouvernance reposant sur le PPP et un accès au programme de porteurs de projets atypiques existe donc, mais il est loin d'être « automatique » et dépend de la façon dont cette nouvelle gouvernance est mise en œuvre.

\subsection{Qui est elle-même une source d'innovation dans les projets financés sur le territoire}

Nous pouvons enfin dans une troisième étape nous intéresser à présent au caractère plus ou moins innovant des projets portés par les bénéficiaires des programmes LEADER. L'objectivation de ce caractère innovant des projets est une question méthodologiquement épineuse pour deux raisons essentielles : (1) il n'existe pas de définition claire et stabilisée d'un projet de développement local innovant, et (2) ce caractère innovant est de toute évidence «relatif » en fonction du territoire dans lequel ou se situe (un projet peut être très innovant dans un territoire ou un pays donné et moins innovant dans le territoire ou le pays voisin). Nous nous appuierons par conséquent sur des appréciations subjectives du caractère innovant des projets, en combinant les appréciations des porteurs de projets eux mêmes (approche déclarative) et les appréciations des acteurs impliqués dans la mise en œuvre des 
programmes (approche experte). Nous ferons par ailleurs l'hypothèse que si on les applique à un nombre important de projets, ces appréciations subjectives donnent une vision relativement robuste de la distribution du caractère innovant des projets.

Le graphique ci-après donne les résultats de cette analyse en ce qui concerne l'approche déclarative (appréciation du caractère innovant de leur projet par les porteurs de projet eux-mêmes). Il montre clairement que les projets portés par certains porteurs de projets atypiques (associations) sont considérés comme nettement plus innovants que ceux portés par les porteurs de projets plus habitués aux financements européens (collectivités, agriculteurs). On note néanmoins que ce résultat ne concerne pas le deuxième type de porteur de projet atypique, les particuliers.

Enfin, même s'il est difficile de le vérifier de façon très précise, certains éléments nous donnent à penser que cette appréciation des porteurs de projets est globalement cohérente avec celle demandée aux acteurs de la mise en œuvre des programmes. En effet, ce sont les acteurs des territoires dans lesquels le nombre de bénéficiaires associatifs était le plus élevé qui ont globalement jugés le plus favorablement le caractère innovant des projets.

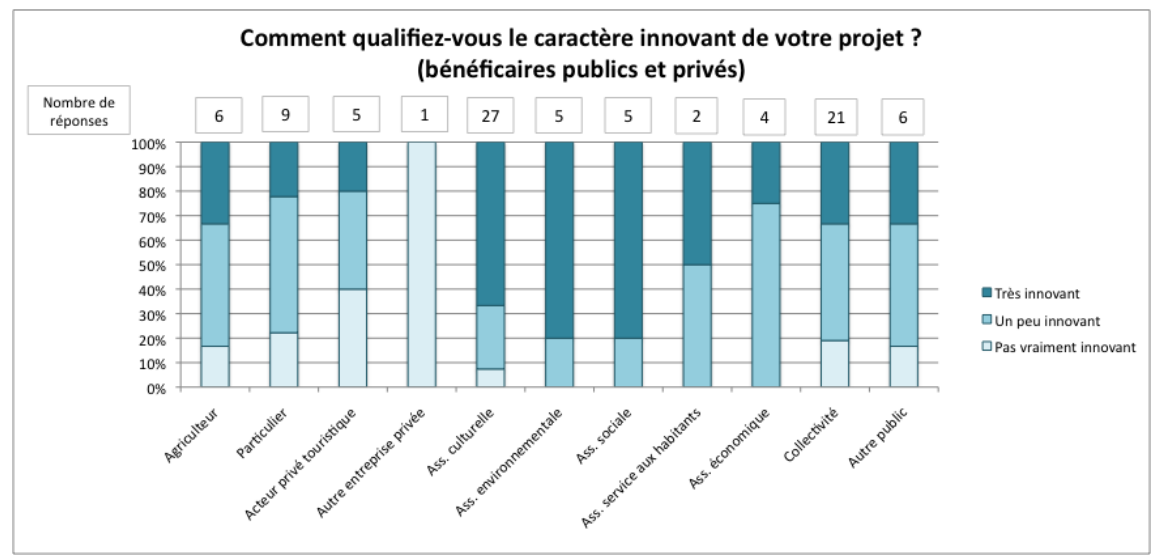

Figure 3 : Comment qualifiez-vous le caractère innovant de votre projet ? (bénéficiaires publics et prives)

Là encore, il convient de rappeler que les auteurs spécialistes de LEADER cités plus haut considèrent également que ce programme a considérablement perdu sa capacité à générer de l'innovation (Doré, 2011; Vollet, 2017). Dans la mesure où ils attribuent notamment ce recul à une évolution de la nature des porteurs de projets (davantage de porteurs de projets publics, moins de porteurs de projets associatifs), ce résultat est tout à fait cohérent avec notre analyse.

\section{Discussion et perspectives}

$\mathrm{Au}$ terme de notre analyse il apparaît donc que la mise en place d'une nouvelle gouvernance territoriale, associant davantage les acteurs privés, peut générer de l'innovation, que ce soit dans la gouvernance elle-même ou dans les projets qui se développent au sein du territoire. Il apparaît également que cet effet n'est pas du tout automatique, et dépend fortement des modalités de mise en place de cette nouvelle gouvernance. Si l'on en revient alors au développement territorial durable, la perspective ouverte par ces résultats peut se 
résumer ainsi: cette nouvelle gouvernance plus ou moins innovante confère-t-elle un caractère (plus) durable au développement territorial ? Cette perspective peut notamment être discutée à partir de deux questions, que nous développons brièvement ci-après :

- les innovations organisationnelles observées (comme la gouvernance publique/privée) peuvent-elles s'ancrer durablement dans les territoires ou restent-elles dépendantes des injonctions via les politiques publiques et/ou de la présence de figures locales ?

Cette question se pose évidemment à la lumière de l'exemple étudié ici, puisque LEADER constitue comme nous l'avons dit une puissante incitation / injonction à mettre en œuvre une gouvernance publique/privée. Elle émerge également de nos observations qui nous ont permis d'apprécier combien, à cette échelle territoriale, le succès — ou l'échec de nombreuses démarches étaient liées à la présence de figures locales. Si ces deux éléments restent de puissants moteurs de la mise en œuvre de nouvelles gouvernances, certains travaux semblent montrer que la «capacité» des territoires à produire de l'innovation, à résister aux chocs, à s'adapter aux évolutions de contexte peut d'une part « s'internaliser » ou «s'incorporer » dans les territoires eux-mêmes, et d'autre part augmenter avec le temps, le cas échéant grâce au soutien de politiques publiques conçues à cet effet. Nous pensons ici aux travaux menés, notamment par la région Nord Pas de Calais ${ }^{17}$, sur la résilience territoriale, ainsi qu'aux travaux sur l'ingénierie « incorporée » (Barral et al., 2010). Ces travaux semblent particulièrement importants pour le champ de la gouvernance territoriale et nécessiteront d'être mis en œuvre dans le temps long pour apporter des réponses à cette première question soulevée ici.

- la participation accrue des acteurs privés dans la gouvernance, et plus largement de la société civile, se traduit-elle par un infléchissement des trajectoires de développement vers davantage de durabilité ?

Cette deuxième question est évidemment trop complexe pour être traitée ici en profondeur. Mais elle peut être éclairée à l'aune des nombreux résultats des travaux d'évaluation menés sur la démarche LEADER, qui soulignent notamment, et de façon robuste: (1) un effet positif de LEADER sur l'attractivité territoriale, (2) un effet d'entraînement sur d'autres sources de financement, (3) un effet sur la création de réseaux, susceptible d'améliorer le capital social du territoire. Si l'on considère que ces trois effets participent de la durabilité, on a alors un exemple de lien entre gouvernance renouvelée et développement territorial durable.

\section{Références bibliographiques}

1. Barbut L., Desgree A., Muhlberger S., 2014. Quelle contribution des Parcs Naturels Régionaux au développement durable de la métropole parisienne?, communication présentée au colloque Dimitrie Cantemir de l'Université de Iasi.

2. Barral F., Thumerel B., Simoulin V., 2010. Ingénierie de projet et excellence territoriale, De nouvelles fabriques de territoires, 2010.

3. Bertrand N., Moquay P., 2004. La gouvernance locale, un retour à la proximité, Economie Rurale ${ }^{\circ} 280$, pages $77-95$.

${ }^{17}$ Aujourd'hui « Hauts de France » depuis la réforme territoriale de fin 2015. 
4. Carriere Jp., Hamdouch A., Iatu C., 2016. Développement durable des territoires, Economica.CHIA E. et al., "Comment analyser la gouvernance territoriale ? Mise à l'épreuve d'une grille de lecture », Colloque AISRE-ASRDLF Aoste (Italie), 2010

5. Cour des Comptes Européenne, 2010. Mise en æuvre de l'approche LEADER pour le développement rural, Rapport spécial $\mathrm{n}^{\circ} 5$.

6. DIACT-ARF, 2009. Référentiel d'évaluation de l'innovation sociétale et territoriale.

7. Dore G., 2011. Le programme européen LEADER a 20 ans : bilan et perspectives à partir du cas français, 5èmes journées de Recherches en Sciences Sociales, Agrosup Dijon,

8. EPICES, 2014.Réalisation et appui méthodologique de l'évaluation finale du programme Leader 2007 - 2013 en Région Champagne-Ardenne.

9. Leloup F., Moyart L., Pecqueur B., 2005.La gouvernance territoriale comme nouveau mode de coordination territoriale ?, Géographie, économie et société 2005/4 (Vol.7), 321-332.

10. Mathe J., Vollet D.,2014. Evaluation régionale LEADER en Auvergne : un bilan mitigé et contrasté en termes de valeur ajoutée, Sciences Eaux et Territoires ,13.

11. Theys J., 2002. La gouvernance, entre innovation et impuissance, Développement durable et Territoires, dossier $\mathrm{n}^{\circ} 2$.

12. Vollet D., Bosc C., 2017. Mesure de la performance des politiques européennes de développement rural par l'estimation de leur valeur ajoutée territoriale : application au programme LEADER du Pays d'Aurillac, Revue d'Economie Rurale et Urbaine.

13. Xhauflair V., Pichault F., Maesschalck M.F., 2010. Partenariats inter-organisationnels et nouvelles formes de gouvernance : les conditions d'un compromis équilibré et pérenne, Management \& Avenir ,33, 298-316. 